\title{
Modeling Blood Pulsatile Turbulent Flow in Stenotic Coronary Arteries
}

\author{
Violeta Carvalho MEtRICs \\ Research Center \\ University of Minho \\ Guimarães, Portugal \\ violeta.carvalho@dem.umi \\ nho.pt
}

\author{
Nelson Rodrigues \\ ALGORITMI Research \\ Center \\ University of Minho \\ Guimarães, Portugal \\ nelson.rodrigues@dps.umi \\ nho.pt
}

\author{
Rui A. Lima \\ MEtRICs Research \\ Center \\ University of Minho \\ Guimarães, Portugal \\ rl@dem.uminho.pt
}

\author{
Senhorinha Teixeira \\ ALGORITMI Research \\ Center \\ University of Minho \\ Guimarães, Portugal \\ st@dps.uminho.pt
}

Received: June 2, 2020. Revised: September 18, 2020. Accepted: October 14, 2020. Published: October 20, 2020.

\begin{abstract}
Atherosclerosis is a potentially serious illness where arteries become clogged with fatty substances called plaques. Over the years, this pathological condition has been deeply studied and computational fluid dynamics has played an important role in investigating the blood flow behavior. Commonly, the blood flow is assumed to be laminar and a Newtonian fluid. However, under a stenotic condition, the blood behaves as a non-Newtonian fluid and the pulsatile blood flow through coronary arteries could result in a transition from laminar to turbulent flow condition. The present study aims to analyze and compare numerically the blood flow behavior, applying the k- $\omega$ SST model and a laminar assumption. The effects of Newtonian and non-Newtonian (Carreau) models were also studied. In addition, the effect of the stenosis degree on velocity fields and wall shear stress based descriptors were evaluated.

According to the results, the turbulent model is shown to give a better overall representation of pulsatile flow in stenotic arteries. Regarding, the effect of non-Newtonian modeling, it was found to be more significant in wall shear stress measurements than in velocity profiles. In addition, the appearance of recirculation zones in the $50 \%$ stenotic model was observed during systole, and a low TAWSS and high OSI were detected downstream of the stenosis which, in turn, are risk factors for plaque formation. Finally, the turbulence intensity measurements allowed to distinguish regions of recirculating and disturbed flow.
\end{abstract}

Keywords - atherosclerosis, CFD, coronary arteries, hemodynamics, non-Newtonian, turbulence.

\section{INTRODUCTION}

Cardiovascular diseases (CVDs) are the leading cause of death globally, and it is expected that by 2030 they will cause over 22 million deaths per year. Atherosclerosis is the main underlying pathological process of CVDs, and it consists of an inflammatory disorder induced by several unhealthy behaviors and lifestyles, that causes the narrowing of the arteries' lumen [1]. During early stages, there are no significant changes in the flow dynamics, nevertheless, as time progresses, these plaques play a critical role in altering the flow characteristics and transition to turbulence can occur, even at much lower Reynolds number [2], [3]. To understand the flow field changes, several hemodynamic studies have been conducted [4], [5]. This can be achieved under in vitro conditions, by performing flow visualizations through particle tracking methods, or under in vivo conditions by using different measurement techniques, such as MRI or Doppler ultrasound [6]. However, in both cases is difficult to measure with enough accuracy some hemodynamic parameters, e.g wall shear stress (WSS). For this reason, computational models have been used as an auxiliary tool for obtaining more reliable results [7]-[9]. In particular, fluidstructure interaction (FSI) [10] and computational fluid dynamics (CFD) [4] have been used as an effective alternative to investigate in detail several blood flow phenomena in blood vessels and an important component in the study of CVDs, allowing the calculation of various hemodynamic parameters with high precision [2], [11], [12].

One of the earliest detailed studies of the flow in idealized stenotic models was conducted by Young [13]. Nevertheless, over the past few decades, the investigation into hemodynamics has grown exponentially and researchers have taking different assumptions, because of the blood flow complexity. Some authors assumed the blood flow in coronary arteries to be laminar [4], [14]-[19] and behave as Newtonian fluid [17], [20]-[23]; others studied turbulence transition [2], [3], [24]-[26] and non-Newtonian effects [2], [5], [15], [21], [27]. In fact, in large vessels, blood can be considered as a homogeneous fluid with constant viscosity. However, in the presence of stenosis, it is observed that the viscosity decreases with increasing velocity and reduction of vessel diameter. Therefore, the non-Newtonian behavior should be considered [18]. Another important factor to the local hemodynamics is the shape of the stenosis. Some researchers apply idealized stenosis shapes [23], [27], [28], while others make use of artery stenotic models based on medical images [14], [19], [29]. In a recent study, Wong et al. [30] studied both idealized and patient-specific coronary arteries and showed how the curvilinearity and narrowing complications affect the computed WSS, associated with the risk of atherosclerosis.

Moreover, in hemodynamic studies, it is also important to study the WSS based descriptors - time-averaged wall shear stress (TAWSS), and oscillatory shear index (OSI) [31]. These are important tools to predict disturbed flow conditions, to estimate the development of local atherosclerotic plaques, and also to understand atherosclerosis progression [32]. Indeed, it has recently been shown that both high WSS as well as low and, in particular, oscillatory WSS can play an important role in these processes [33]. For instance, Gaudio et al [18] compared the Newtonian and non-Newtonian Carreau-Yasuda model in patientspecific stenotic coronary arteries with different degrees of stenosis. For that, the researchers investigated numerically the blood velocity and the distribution of the shear stress indices at different times of the cardiac cycle. Their results showed that WSS and TWASS for the Carreau-Yasuda model are slightly higher when compared to the Newtonian model. In another study, Carvalho et al [34] applied the Carreau nonNewtonian model and also investigated the WSS indices in different degrees of stenosis. They observed that the WSS and TAWSS are maximum in the stenosis throat which is in agreement with the previously mentioned study of Gaudio and colleagues. Kamangar and his team [29] investigated the 
effect of stenosis in left coronary arteries on the hemodynamics in suspected diseased patients, using nonNewtonian (Carreau model) and laminar assumptions. They observed a considerable pressure drop and an increase in the velocity and wall shear stress at the stenosis throat. In the post stenotic section, they observed the prevalence of a recirculation zone which can result in the growth or formation of new stenosis.

To understand the turbulence transition effect on hemodynamic parameters, Mahalingam et al. [2] applied the SST k- $\omega$ model during pulsatile flow through coronary arteries for different degrees of stenosis. They demonstrated that the transition to turbulence starts occurring above $50 \%$ stenosis and becomes fully turbulent above and beyond $70 \%$ of stenosis. Another interesting study was conducted by Moreno \& Bhaganagar [3] in which different types of stenosis were evaluated based on real data, and also used the SST k- $\omega$ model. They observed that the presence of turbulence and the location of transition from laminar to turbulence state are additional hemodynamic parameters to identify plaques vulnerable to rupture. According to the literature, the best suited turbulent model to simulate the blood flow in pulsatile conditions is the transitional $\mathrm{k}-\omega$ model, also referred as SST $\mathrm{k}-\omega$. This has been investigated and validated by several authors [3], [25], [26], [35] using as reference the experimental study of Ahmed and Giddens [36].

However, the comparison between laminar and turbulent modeling has not been greatly investigated and the selection of the proper models to simulate the blood flow is vital to ensure the accuracy and reliability of numerical results. This is of great importance, since the objective of hemodynamic numerical studies is to simulate as best as possible the phenomena that occur in vivo.

In summary, from the aforementioned reviewed literature, it can be observed that the use of non-Newtonian models to simulate blood flow has grown, as well as, the use of turbulence models, being the Carreau and SST k- $\omega$ models, the most widely used to study stenotic arteries. However, the impact of the models' selection on results has not been deeply studied.

In this regard, in the current study, an analysis of the turbulence modeling effects on blood flow is performed, comparing the SST k- $\omega$ model to a laminar flow. The effect of shear-thinning blood behavior was also studied by using the Carreau model. For this purpose, an idealized coronary artery with $70 \%$ of stenosis was developed since when coronary arteries are affected by atherosclerosis can lead to a heart attack [37]. Additionally, the effect of stenosis severity was also evaluated both in velocity and in WSS based indicators. Another hemodynamic factor with an indirect association with thrombotic activity is the level of turbulence intensity (TI) and, thus, it was also computed. With the present study, important outputs were obtained, demonstrating the importance of choosing the appropriate turbulence model, and detecting atherosclerosis at an earlier stage of the disease.

\section{Methodology}

\section{A. Geometry of the stenotic model}

The first step required to perform computational simulations of blood flow is to obtain the three-dimensional geometry of the artery's lumen. Taking into account that the coronary arteries were selected for this study, the dimensions were selected carefully from the literature to accurately mimic the real conditions of blood flow in these arteries. The diameter selection was based on real data obtained by Zhou et al. [38] in 2017. Moreover, to develop the stenotic area, it was found that several authors [2], [39], [40] consider the length of the stenosis as twice the normal diameter of the artery. In addition, the entrance length was chosen so that the velocity profiles could be fully established proximal to the stenosis throat.

To perform this step the Design Modeler tool in ANSYS ${ }^{\circledR}$ workbench was used and the final stenotic model developed, and its geometric parameters are represented in Fig.1.

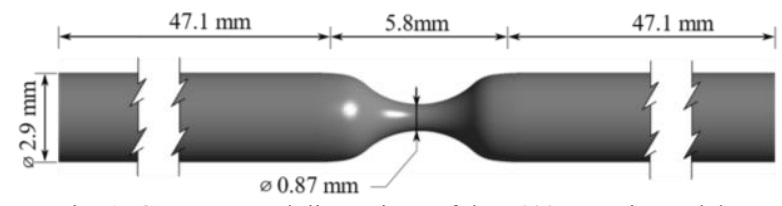

Fig. 1. Geometry and dimensions of the $70 \%$ stenotic model.

\section{B. Mathematical modeling}

Blood flow is modeled using the well known NavierStokes and the continuity equations as follows in Equation 1 and 2,

$$
\begin{gathered}
\nabla \cdot \vec{u}=0 \\
\rho\left(\frac{\partial \vec{u}}{\partial t}+\vec{u} \cdot \nabla\right)=-\nabla p+\mu \nabla^{2} \vec{u}
\end{gathered}
$$

where $\vec{u}$ is the velocity vector, $p$ is the static pressure, $\rho$ is the fluid density, and $\mu$ the dynamic viscosity. These are applied in the presence of a laminar regime, however, when turbulent flows are verified, the use of turbulent models is needed, and some modifications to the momentum equation are included as can be seen in Equation 3:

$$
\frac{\partial \rho \bar{u}_{i}}{\partial t}+\frac{\partial}{\partial x_{j}}\left(\rho \bar{u}_{i} \bar{u}_{j}+\rho \overline{u^{\prime}{ }_{l} u_{J}^{\prime}}\right)=-\frac{\partial \bar{p}}{\partial x_{i}}+\frac{\partial \overline{\overline{\tau_{l j}}}}{\partial x_{j}}
$$

where $\bar{u}_{i}$ and $\bar{u}_{j}$ are the velocity components in the $x_{i}$ and $x_{j}$ direction, $\rho \overline{u^{\prime}{ }_{l} u_{j}^{\prime}}$ are the Reynolds stresses, $\mathrm{p}$ is the pressure, and $\tau_{i j}$ is the mean viscous stress tensor.

Despite the diversity of turbulence models, in the present study, the transitional $k-\omega$ model was selected because of its reliability and accuracy to capture the transition from a laminar to a turbulent flow. This model is similar to the standard model, but with some modifications. The SST $k-\omega$ model has two other transport equations, one for the intermittency and one for the transition onset criteria. This is of interest in this study since the flow is not fully turbulent in the entire domain or throughout the entire cardiac cycle [3], [26]. Moreover, this model is used to effectively combine the robust and accurate formulation of the $k-\omega$ model in the nearwall regions with the freestream independence of the $k-\varepsilon$ model far from the wall [3], [41], [42]. The transport equations for the turbulent kinetic energy, $k$, and the specific dissipation rate, $\omega$ are modeled as follows in Equations 4 and 5.

$$
\begin{gathered}
\frac{\partial}{\partial t}(\rho k)+\frac{\partial}{\partial x_{i}}\left(\rho k u_{i}\right)=\frac{\partial}{\partial x_{j}}\left(\Gamma_{k} \frac{\partial_{k}}{\partial x_{j}}\right)+G_{k}-Y_{k}+S_{k} \\
\frac{\partial}{\partial t}(\rho \omega)+\frac{\partial}{\partial x_{i}}\left(\rho \omega u_{i}\right)=\frac{\partial}{\partial x_{j}}\left(\Gamma_{\omega} \frac{\partial_{\omega}}{\partial x_{j}}\right)+G_{\omega}-Y_{\omega}+D_{\omega}+S_{\omega}
\end{gathered}
$$


$G_{k}$ represents the generation of turbulence kinetic energy due to mean velocity gradients, $G_{\omega}$ represents the generation of $\omega . \Gamma_{\omega}$ and $\Gamma_{k}$ represent the effective diffusivity of $k$ and $\omega$, respectively. $Y_{k}$ and $Y_{\omega}$ represent the dissipation of $k$ and $\omega$ due to turbulence. $D_{\omega}$ represents the cross-diffusion term. $S_{k}$ and $S_{\omega}$ are user-defined source terms.

\section{Blood flow assumptions}

In this study, both Newtonian and non-Newtonian models were studied in order to conclude if there are significant differences in the results obtained. When the blood is modeled as a Newtonian fluid, appropriate rheological parameters were applied with a density of $1072.033 \mathrm{~kg} \cdot \mathrm{m}^{-3}$ and a viscosity of $0.00315 \mathrm{~Pa} \cdot s$, which are similar to those used in previous studies [4], [14], [43]. For the pulsatile nonNewtonian blood flow simulations, the selected viscosity model was the Carreau model, since it is commonly used by several authors [14], [15], [44] to simulate the shear-thinning blood behavior and it is considered the most suitable to perform these simulations. In the Carreau model, the viscosity $(\mu)$ is given by Equation 6:

$$
\mu=\mu_{\infty}+\left(\mu_{0}-\mu_{\infty}\right)\left[1+\lambda \dot{\gamma}^{2}\right]^{\frac{n-1}{2}}
$$

where, according to the previously cited authors, $\mu_{\infty}=$ $0.00345 \mathrm{~Pa} \cdot s$ is the infinite shear viscosity, $\mu_{0}=$ $0.0560 \mathrm{~Pa} \cdot s$ is the blood viscosity at zero shear rate, $\dot{\gamma}$ is the instantaneous shear rate, $\lambda=3.313 s$ is the time constant and $n=0.3568$ is the power-law index.

\section{Boundary conditions}

The appropriate boundary conditions are critical for the performance of a realistic and accurate simulation. In this investigation, the artery walls were considered rigid by applying a no-slip condition.

In addition, to ensure that the present study reflects the realistic simulation of in vivo conditions, a physiological inlet velocity profile adopted by Doutel et al. [14], as seen in Fig. 2, was applied.

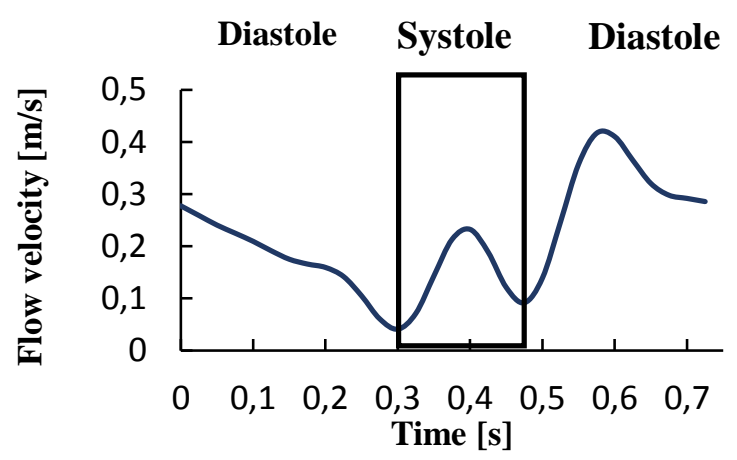

Fig. 2. Velocity profile adopted in the simulation, representing a full cardiac cycle, according to Doutel et al. [14].

The mean inlet velocity $\left(V_{m}\right)$ of the cardiac cycle is defined as shown in Equation 7, where $\overline{V_{m}}$ is the average velocity along the cycle, $w$ is the angular frequency of the cardiac cycle, and $a_{i}$ and $b_{i}$ are parameters of the fitting model and they were obtained from [14]. Equation 7 was written in Clanguage using the interface of User Defined Function (UDF) of Fluent and linked with the solver.

$$
V_{m}(t)=\frac{\overline{V_{m}}}{a_{0}}\left\{a_{0}+\sum_{i=1}^{5}\left[a_{i} \cos (i w t)+b_{i} \sin (i w t)\right]\right\}
$$

It should be noted that four consecutive cardiac cycles were run and only the results from the last cycle were analyzed. At the outlet, a zero gauge pressure condition was applied.

\section{E. Mesh generation and optimization}

The numerical meshing and simulations were performed in the computational fluid dynamics (CFD) package ANSYS Fluent (version R1). In Ansys Meshing, a hexahedral mesh was created with a bias factor to ensure the accuracy of computational calculations near the wall, and a more refined mesh in the stenotic section. To assure a mesh independent solution, grid tests were made by refining the mesh and rerunning the simulations until no significant differences were observed. For each mesh, the maximum velocity was calculated, and the results are represented in Fig. 3.

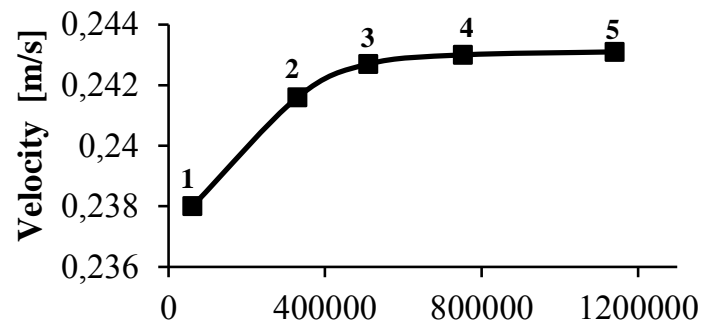

Number of elements

Fig. 3. Variation of the velocity magnitude with mesh refinement for with $70 \%$ of stenosis.

It can be concluded that for meshes with more than 500000 elements, the results are independent of the mesh. Since the differences are minimal between the results of mesh 3 and 4 , and taking into account the computational time, mesh 3 was selected, with 510,720 elements (Fig.4).
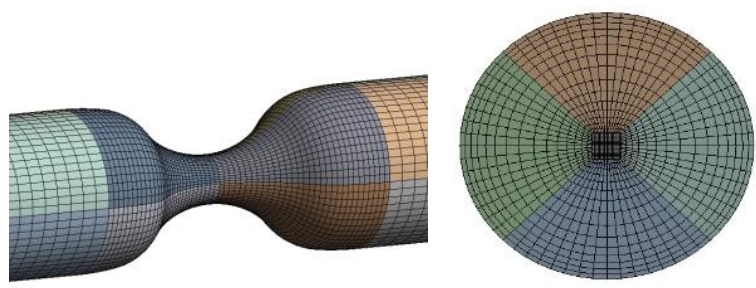

Fig. 4. Optimized mesh obtained for the $70 \%$ stenotic model.

\section{F. Numerical solution}

In Fluent, the finite-volume discretization method is applied. In this method, the integral form of the conservation equations is used as the starting point. Moreover, the solution domain is subdivided into a finite number of contiguous control-volumes, the conservation equations are applied to each control-volume and, finally, a system of algebraic equations for the variables at some set of discrete locations in space and time is obtained, that can be solved numerically [45]. However, since the discretized equations are non-linear, an iteration scheme is used to solve them. In the present study, the semi-implicit method for pressure linked equations (SIMPLE) scheme was used, in which, the governing equations are solved sequentially, segregated from one another. Furthermore, by default, ANSYS ${ }^{\circledR}$ Fluent stores discrete values of the scalar at the cell centers. However, since the values of the variable on the faces are not known, they must be obtained by interpolating the values on the cell center values. In this investigation, the second-order upwind 
scheme was used [41]. Higher-order precision is achieved at cell faces by Taylor's series expansion of the cell-centered solution in relation to its centroid.

\section{G. Post-processing}

The last step of the numerical modeling consists of the analysis of the results obtained. ANSYS software has different methods such as contour plot, vector plot, data curves and streamlines. Using these different ways, the calculated values of the main variables, pressure, velocities and turbulent parameters can be confirmed, along with their precision. Besides that, other parameters have been studied from the results. Over the years, it has been proved that the WSS indices are related to lipid accumulation and, thus, these are held as important risk-factors in coronary artery disease.

For incompressible fluids, spatial WSS, $\tau_{w}$, is calculated by Equation 8 , being $\dot{\gamma}$, the deformation rate.

$$
\tau_{w}=\mu \frac{\partial u}{\partial y}=\mu \dot{\gamma}
$$

Besides the WSS, in the present study, two other indicators, TAWSS and OSI, are considered in order to assess the possibility of development of new stenosis, as well as the plaque rupture. The TAWSS is the time-averaged magnitude of the WSS and is used to evaluate the total shear stress exerted on the wall during a cardiac cycle [32]. This is calculated by applying Equation 9:

$$
T A W S S=\frac{1}{T} \int_{0}^{T}|W S S| d t
$$

It has also been observed that oscillatory WSS promotes atherosclerosis [31], [33]. However, this information is not given by the TAWSS. To account for such effect, the oscillatory shear index, OSI, was introduced and it is calculated by Equation 10:

$$
O S I=\frac{1}{2}\left(1-\frac{\left|\int_{0}^{T} W S S d t\right|}{\int_{0}^{T}|W S S| d t}\right)
$$

The OSI varies between 0 and 0.5 . When OSI is equal to 0 , the flow is unidirectional. When OSI is equal to 0.5 , it means that the WSS field is fully oscillatory [33].

Furthermore, turbulence intensity (TI) is also an important parameter for predicting thrombus formation and plaque rupture and, therefore, it is of clinical significance to investigate it [46]. The turbulence intensity, TI, is defined as the ratio of the root-mean-square of the velocity fluctuations, $u^{\prime}$, to the mean flow velocity, $u_{a v g}$, as shown in Equation 11 [41]:

$$
T I \equiv \frac{u^{\prime}}{u_{\text {avg }}}
$$

\section{RESULTS AND DISCUSSION}

\section{A. Turbulent model and laminar comparison}

To assess the flow behavior through the simulated stenotic artery, a velocity profile of the fluid flow was plotted along the geometry center of the $70 \%$ stenotic model. The plotted data starts near the stenosis center, where $X=0$ and follows the blood flow direction until $X=-50 \mathrm{~mm}$. Fig. $5 \mathrm{I}$ ) and II) represent the velocity flow in the diastolic and systolic peak, respectively, assuming the blood as a Newtonian fluid.
For both phases, the effect of the laminar and turbulent behavior according to the SST $k$ - $\omega$ model was studied.

I)

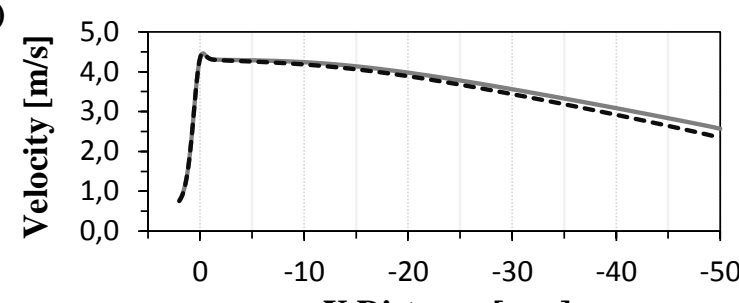

II)

X Distance [mm]

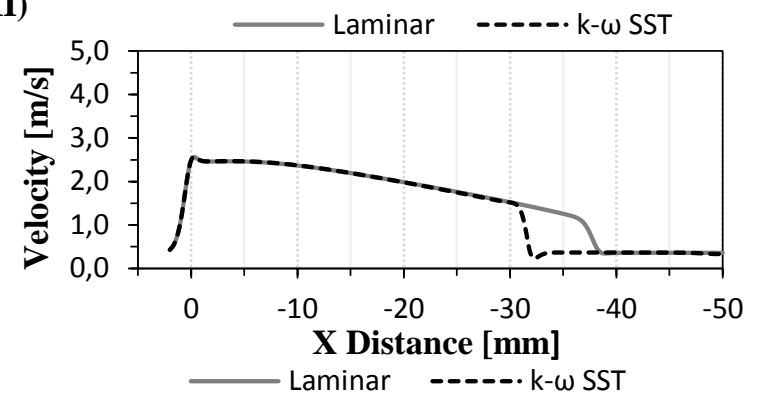

Fig. 5. Simulated centerline velocity at the time of the: I) diastolic peak; II) systolic peak of the input velocity profile.

Through observation of Fig. 5, it is possible to observe that the velocity waveform near and at the stenosis throat $(\mathrm{X}=0)$ the predicted values by both models in the different phases of the cardiac cycle are in excellent agreement. However, after the contraction, the differences are more notable.

In the diastolic peak (Fig. $5 \mathrm{I}$ ), the SST $k-\omega$ and laminar model show a similar behavior along the geometry, with a gradual reduction in velocity after stenosis. These results are in good agreement with a study conducted by Straatman et al. [26], who also compared the SST $k-\omega$ model with experimental data obtained by Ahmed and Giddens [36]. Looking at Fig. 5 II, it can be also observed an identical behavior for both models, however, the transitional $\mathrm{k}-\omega$ model presents an earlier drop in velocity, that marks the end of the recirculation zone. The laminar model predicts a slightly higher recirculation length. In Straatman work [26], it was also showed that the transitional $k-\omega$ model overpredict the length of the recirculation when compared to the experimental data obtained by Ahmed and Giddens [36]. Taking into account this observation and the results obtained in Fig.5 II, it can be concluded that the laminar model overpredicts considerably more the recirculation length observed experimentally. Therefore, it seems that the laminar model is not the most adequate to simulate the blood flow in stenotic arteries.

Despite the few differences between the two models, it was showed that the model selection influences the results and the most adequate model seems to be the transitional $k$ $\omega$. For this reason, the remaining simulations were performed with that model.

\section{B. Newtonian and Non-Newtonian behavior}

To compare the non-Newtonian effects in flow behavior, the velocity profiles in the stenotic and post stenotic section $(\mathrm{X}=-2.5 \mathrm{~mm})$, only at the diastolic peak were evaluated for the $70 \%$ stenotic model (Fig. 6). 
a)

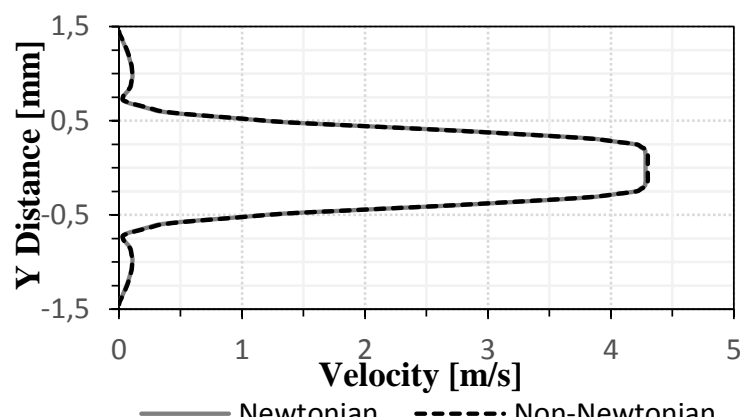

b)

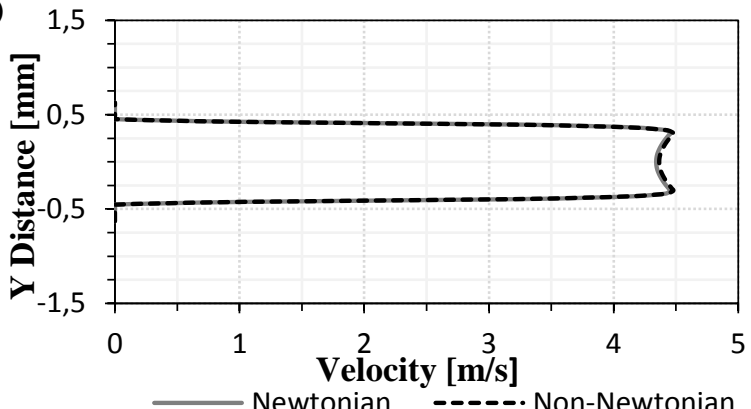

Fig. 6. Comparison between the velocity profiles at: a) stenotic section; b) the post-stenotic section.

As it can be observed in Fig. 6 a) and b), the two models present the same behavior and there is only a small variation in the maximum velocity. It can be seen a lower maximum value in the curve of the Newtonian model compared to the Carreau model.

In addition, to evaluate if the differences are significative a statistical analysis was performed by applying a t-test for a significance level, $\alpha$, of 0.1 . The $\mathrm{P}$ values obtained were approximately 0.99 for both cases, Fig. 6 a) and b). This shows that there are not significant differences between the two models.

Fig. 7 shows the wall shear stress (WSS) along the coronary artery, in particular in the region of the stenosis at the diastolic peak.

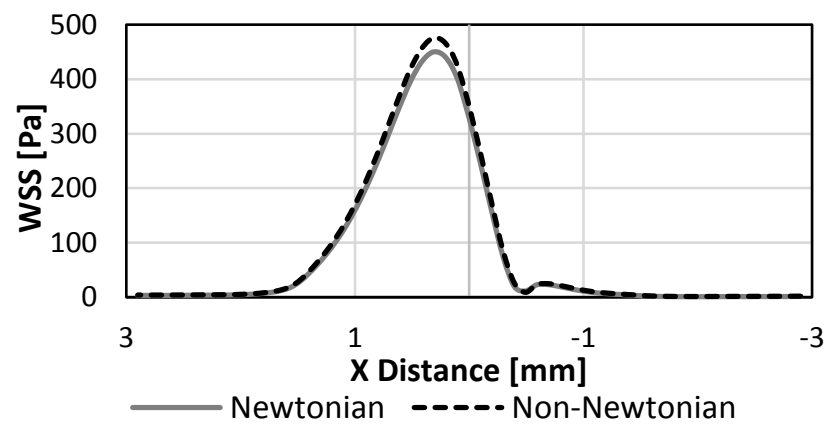

Fig. 7. Comparison between the WSS using the Newtonian and Carreau model.

The maximum value of WSS is located in the maximum narrowing area and the differences between the two models are more perceptible than in velocity. Comparing the Newtonian model to the Carreau model, the first one predicts a lower value of WSS, visible in the peak where there is the presence of the stenosis. These results are in agreement with a previous study conducted by Gaudio et al. [18].
Similarly to the previous results, the t-test was applied in the same conditions, and a $\mathrm{P}$ value of 0.82 was obtained. As before, according to the t-test, the differences are not considered significant. However, there is a reasonable difference between the two $\mathrm{P}$ values observed, which proves the bigger impact of non-Newtonian assumptions on shear stress measurements.

\section{Effect of stenosis severity}

Taking into account the prior results, the Carreau and the SST k- $\omega$ models were applied to investigate the effect of stenosis severity. For this purpose, two additional cases were simulated, a stenotic model with $50 \%$ of diameter reduction and a healthy model. Note that, for these models, similar meshes to the optimized one showed previously, were made.

To evaluate the flow behavior through the stenotic artery in the presence of different degrees of stenosis $(0,50$ and $70 \%$ ), the centerline velocity was plotted and the results obtained are depicted in Fig.8 (a) and (b), corresponding respectively, to the systolic and diastolic phases of the cardiac cycle.

In both phases of the cardiac cycle, the stenotic models attain their maximum velocity in the center of the geometry $(\mathrm{X}=0 \mathrm{~mm})$, and as expected, the healthy model maintains its velocity along axis (approximately $0.3 \mathrm{~m} / \mathrm{s}$ during systole and $0.6 \mathrm{~m} / \mathrm{s}$ during diastole). Moreover, both stenotic models present a similar behavior until the stenosis throat. Nevertheless, the differences after the contraction are quite significant.

During systole (Fig.8 (a)), it can be observed that for both models there is a continuous drop in the centerline velocity after stenosis, until afterward occurs a more noticeable drop in velocity, which is more accented in the $70 \%$ stenosis case. This marks the end of the recirculation zone predicted by both models. However, the $70 \%$ stenotic model presents higher velocities and a recirculation zone longer than the 50\% model, due to the greater diameter reduction seen in this model.

Similarly to the systolic phase, during diastole (Fig. 8 (b)), the 50\% stenotic model shows a recirculation length significatively lower, marked by an earlier and smoother drop in velocity. In turn, the $70 \%$ stenotic model presents a gradual reduction in velocity until the end of the artery. This is due to the recirculation zone length, which is higher than the channel length. Regarding this result, a remark must be made. Through the analysis of velocity vectors results, the existence of vectors in the opposite direction to the flow was verified since the end of the artery. To confirm that this was a valid result, a new test was performed using the same geometry with a longer length and, indeed, the end of recirculation was observed. This proves that in the presence of this pathology, changes in blood flow can be huge, especially during diastole, where the highest velocities are reached. 
(a)
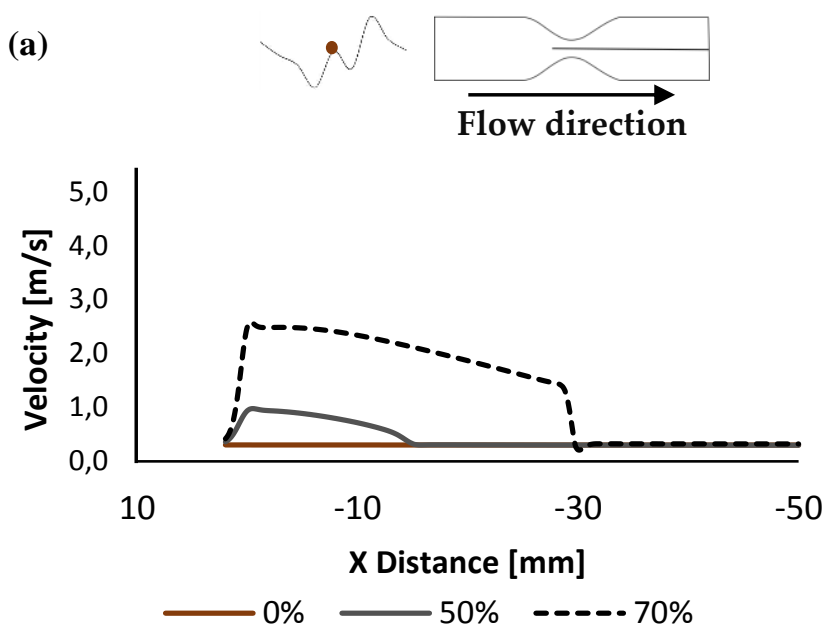

(b)
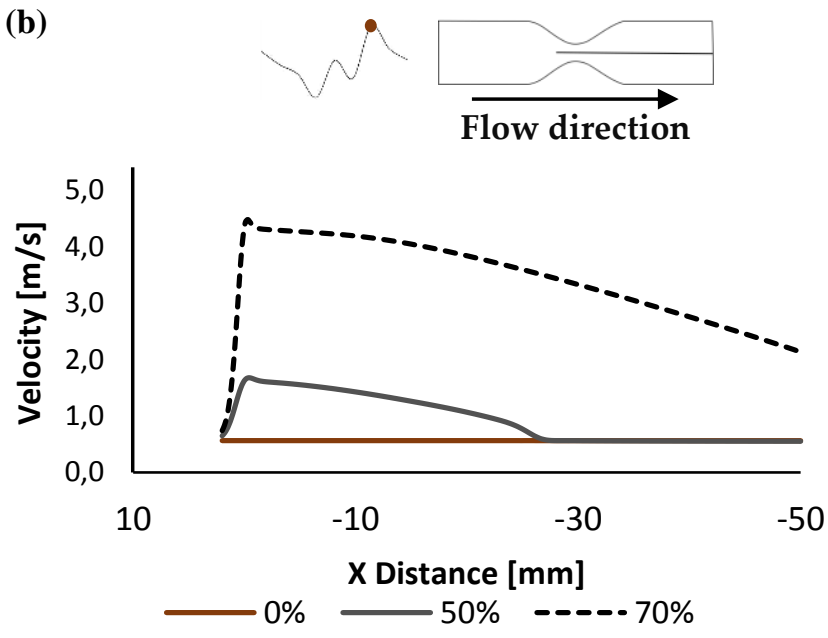

Fig. 8. Centerline velocity at the time of the: (a) systolic peak; (b) diastolic peak of the input velocity profile.

\section{Effect of stenosis on WSS and its indices}

Additionally, as WSS is an important factor in the development of atherosclerosis, its evolution along the stenotic artery was also studied in the two main phases of the cardiac cycle, systole, and diastole (Fig.9 (a) and (b), respectively).

Regarding the WSS results during systole (Fig.9 (a)), for $\mathrm{X} \approx-30 \mathrm{~mm}$ can be observed a small peak in the $70 \%$ stenotic model, which represents the end of the recirculation zone and thus, after that, the WSS values decrease and maintain a constant value until the end. Although this behavior is not perceptible for the $50 \%$ case, a smoother variation in WSS happens, without any marked peak. This is due to the smoother reduction in velocity (Fig.8 (a)) verified in this model when compared to the $70 \%$ case.

Moreover, the maximum WSS is observed at the stenosis throat and its value increases with the degree of stenosis. For $70 \%$ and $50 \%$ of stenosis, $207 \mathrm{~Pa}$ and $39.6 \mathrm{~Pa}$ of WSS are observed, respectively.

During diastole (Fig. 9 (b)), there is no peak in the grey plot in the post stenotic section. This is explained by the continuity of the recirculation in the $70 \%$ case, as clarified previously. For the $50 \%$ model, the behavior is very similar to that obtained during diastole, only presenting lower WSS values. Regarding the healthy model, this presents a constant value of WSS in each phase (approximately $2.3 \mathrm{~Pa}$ during systole and 4.3 Pa during diastole) and shows that in a healthy situation the WSS should be much lower than the ones verified, showing the impact of the degree of stenosis on some hemodynamic parameters.
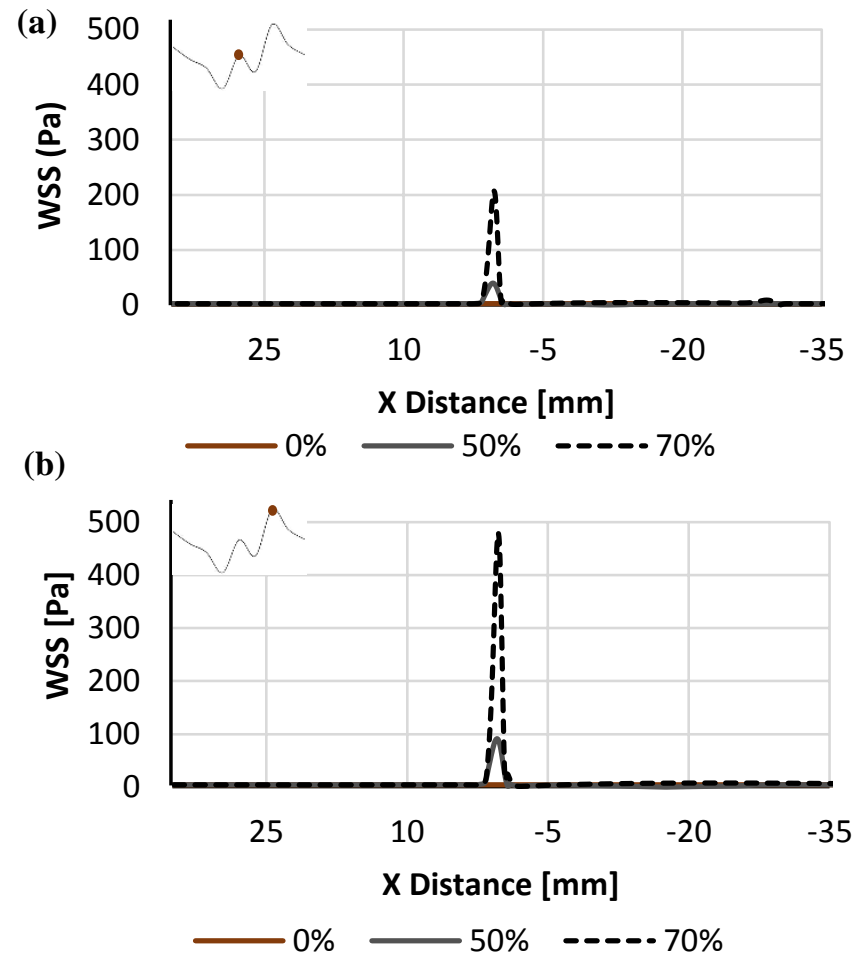

Fig. 9. Comparison between the WSS obtained for each degree of stenosis during (a) systole and (b) diastole.

During diastole, the maximum WSS is also observed at the stenosis throat, however, the values are much higher than during systole. For $70 \%$ and $50 \%$ of stenosis, $480 \mathrm{~Pa}$ and 91.3 $\mathrm{Pa}$ of WSS are observed, respectively.

The results obtained are in agreement with other studies, for instance, Mulani et al. [27] had similar conclusions when comparing a healthy model to stenotic models with a geometry quite similar to those presented in this study. In addition, through the analysis of these results, it can be concluded that effectively the transient condition is extremely important to understand what happens in vivo and comparing both phases of the cardiac cycle, significant differences were observed.

After the WSS was calculated, other hemodynamic parameters including OSI and TAWSS were determined and the results are presented in Fig. 10.

By observing Fig.10 a) and b), it can be seen that both plots have quite similar shapes to those presented in Fig.7. Starting with the OSI results, Fig.10 a), the highest values (0.5) are located right after the stenosis throat. This happens because the stenosis creates complex flow patterns with 
vortices appearance causing the increase of OSI [31]. In the remaining geometry, the OSI values are approximately equal to zero, which means that the flow is unidirectional. In other words, the low OSI values at the stenosis throat are due to the small spatial velocity fluctuations verified in that region. However, downstream of the stenosis, the shear-layer is unstable with spatial fluctuations. Note also that both models (50 and 70\%) have the same results, proving that the $50 \%$ model has also regions with backflow, as previously observed.

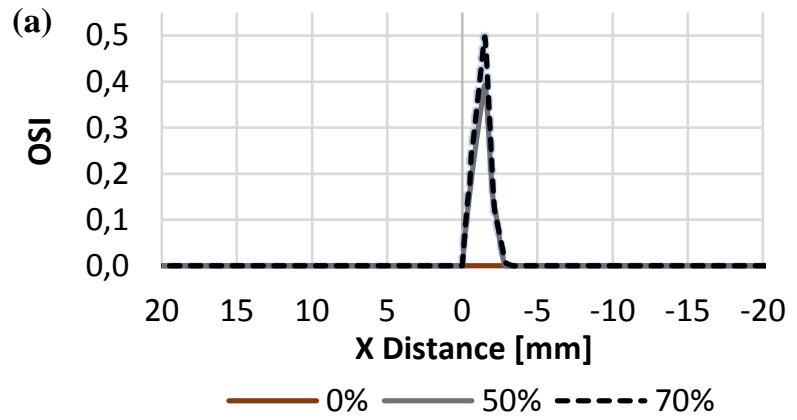

(b)

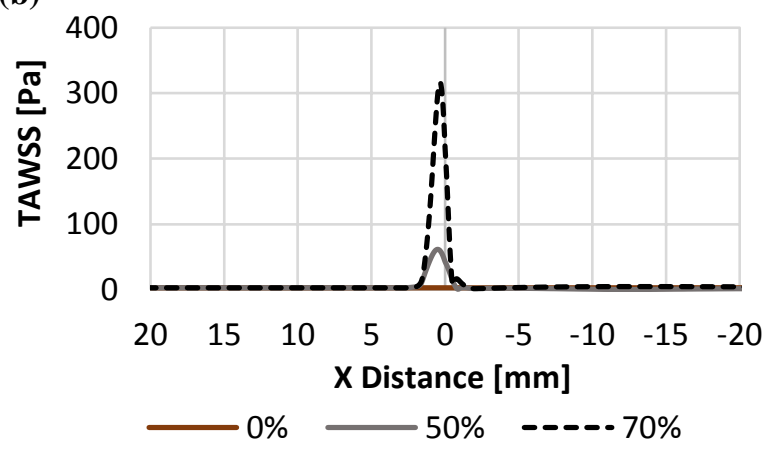

Fig. 10. Comparison between the WSS indices obtained for each degree of stenosis (a) OSI and (b) TAWSS.

On the other hand, the highest values of TAWSS are observed in the stenosis throat. This was expected since this hemodynamic indicator is the time-averaged value of the WSS vector magnitude and, thus, the results presented in Fig.9 and Fig.10 b) are quite similar, however, with lower values of TAWSS, approximately, $300 \mathrm{~Pa}$ for the $70 \%$ stenotic model and $62 \mathrm{~Pa}$ for the $50 \%$ stenotic model [31]. Furthermore, the healthy model $(0 \%)$ shows values close to zero for both indices, showing clearly the hemodynamic impact of atherosclerosis.

Additionally, in general, it can be observed that regions downstream of stenosis had lower TAWSS and higher OSI, which constitute risk factors for the incidence and progression of atherosclerosis [33]. These results are in good agreement with recent studies conducted in arterial stenosis [31], [33], [47].

\section{E. Effect of stenosis on turbulence intensity measurements}

The turbulence intensity is another important hemodynamic parameter that gives a good measure of the instability of the flow [48]. In this study, the turbulence intensity was evaluated during diastole because it is the most critical phase of the cardiac cycle. This was studied for each degree of stenosis in four different axial positions $(x=2.6$ $\mathrm{mm}, \mathrm{x}=0 \mathrm{~mm}, \mathrm{x}=-9 \mathrm{~mm}$, and $\mathrm{x}=-50 \mathrm{~mm}$ ). The results are shown in Fig.11.

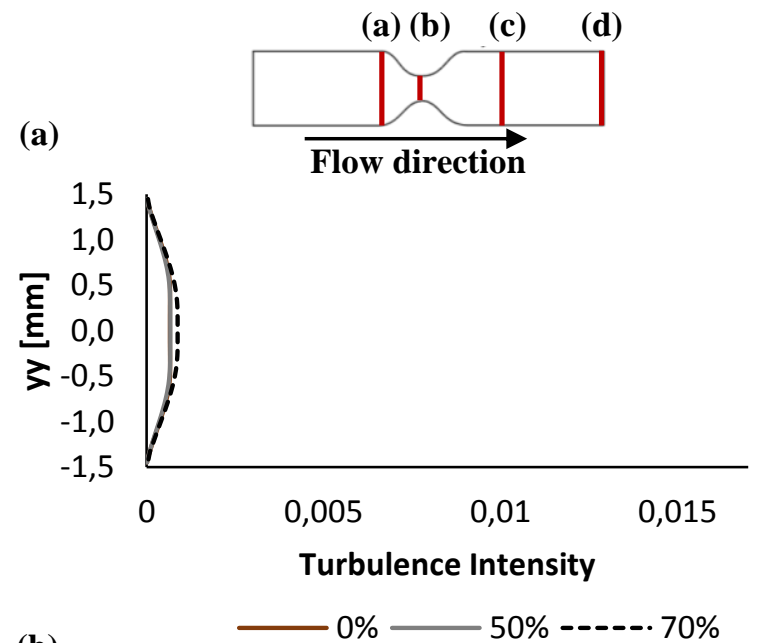

(b)

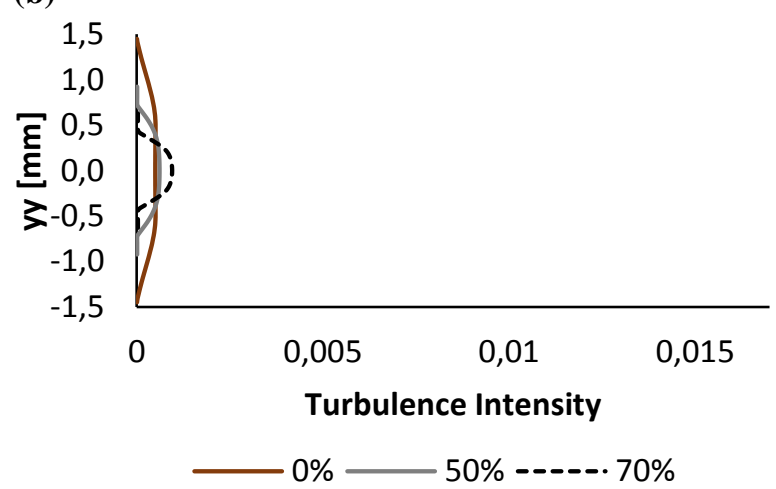

(c)
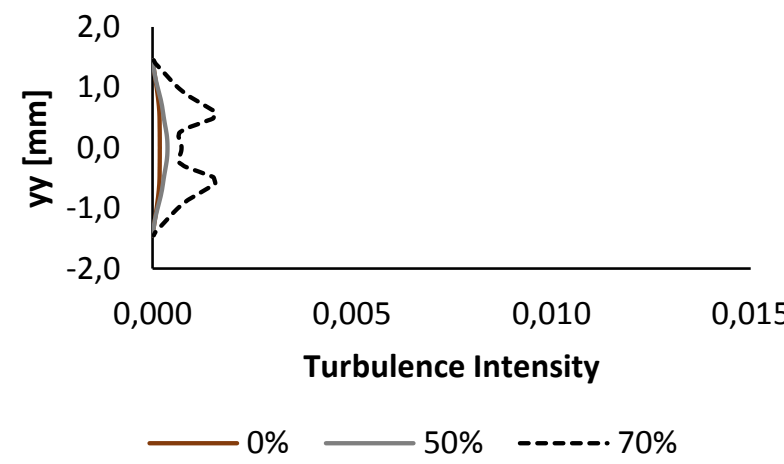

(d)
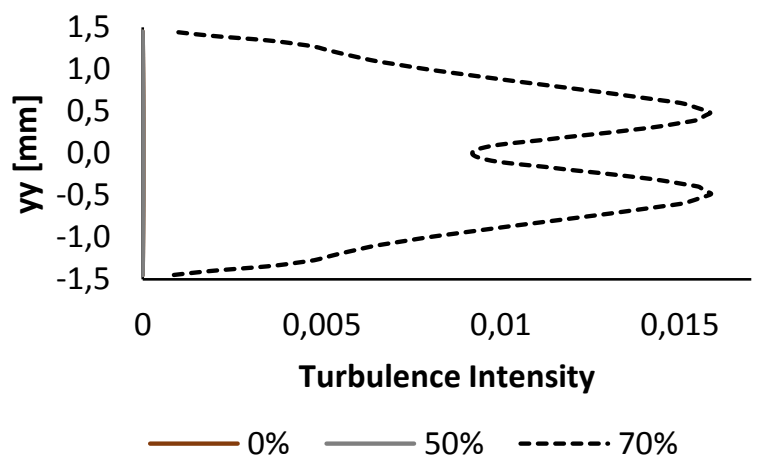

Fig.11. Turbulence intensity profiles at four axial positions: (a) $\mathrm{x}=2.6 \mathrm{~mm}$, (b) $\mathrm{x}=0 \mathrm{~mm}$, (c) $\mathrm{x}=-9 \mathrm{~mm}$, and (d) $\mathrm{x}=-50 \mathrm{~mm}$. 
By observing Fig.11, it can be seen that before stenosis, Fig. 11 (a), the TI of the $70 \%$ stenotic model is slightly higher than the remaining cases which, in turn, present almost the same TI values. Despite those observations, in general, the TI values are very low. Quite similar results are observed in the stenosis throat, Fig. 11 (b), however, although it is not perceptible, the 50\% stenotic model presents higher TI values than the healthy model, as it has been also observed by other authors [46], [49]-[51]. Downstream of stenosis, Fig.11 (c), those differences are more notable and the plot of the $70 \%$ stenotic model presents a different behavior, with a concavity inward in the central zone, and higher values of TI near the wall. This may be explained by the disturbed flow region observed downstream of the stenosis marked by the presence of recirculation areas. Similarly, at the end of the artery, Fig. 11 (d), the highest values of TI are observed for the 70\% stenotic model, which proves the existence of disturbing flows at the outlet, as previously observed in Fig. 8 (b). Moreover, it can also be seen that the other models present TI values close to zero. This pattern clearly indicates that the $70 \%$ stenotic model is the most severe and worrisome case from a clinical point of view.

\section{CONCLUSIONS}

The present study provides new insights on the use of the transitional variant of the $\mathrm{k}-\omega$ model for predicting pulsatile flow through stenotic coronary arteries, instead of the laminar model, which is commonly used by several researchers. Note that, the differences between the two models are verified only in the post stenotic area, where recirculation occurs.

The results from this study also indicate that the nonNewtonian effects are more significant in the wall shear stress measurements, with the Newtonian model predicting slightly lower values in the stenotic section, showing that the selection of the viscosity model changes the results, even in a slightly way. The same tendency was observed in the velocity profiles, however, it is almost imperceptible.

Regarding the effect of stenosis severity, it was shown that the $70 \%$ stenotic model is a severe case presenting longer recirculation zones than the $50 \%$ model and it has also presented the highest WSS values in the stenosis throat, showing the possibility of plaque rupture, i.e, thrombosis. Besides that, other hemodynamic indicators were evaluated and low TAWSS and high OSI were found downstream of stenosis, which, in turn, are also associated with a higher risk of plaque formation. Moreover, the turbulence intensity was found to be useful for characterizing the type of flow and the presence of recirculation zones.

Despite the results agreed with previous studies, further investigations are necessary, since the physiological flows are complex. In this sense, in future work, it would be interesting to validate the numerical results presented in this study by performing in vitro tests to verify the accuracy of these simulations, and also test other turbulence models.

\section{ACKNOWLEDGMENTS}

This work has been supported by FCT - Fundação para a Ciência e Tecnologia within the R\&D Units Project Scope: UIDB/00319/2020, UIDB/04077/2020.

\section{REFERENCES}

Microvascular Dysfunction, First. Springer, 2019.

[2] A. Mahalingam et al., "Numerical analysis of the effect of turbulence transition on the hemodynamic parameters in human coronary arteries," Cardiovasc. Diagnosis Ther., vol. 6, no. 3, pp. 208-220, 2016, doi: 10.21037/cdt.2016.03.08.

[3] C. Moreno and K. Bhaganagar, "Modeling of stenotic coronary artery and implications of plaque morphology on blood flow," Model. Simul. Eng., vol. 2013, no. 7, pp. 1-14, 2013, doi: $10.1155 / 2013 / 390213$.

[4] V. Carvalho, N. Rodrigues, R. Ribeiro, P. F. Costa, R. A. Lima, and S. F. C. F. Teixeira, "3D Printed Biomodels for Flow Visualization in Stenotic Vessels: An Experimental and Numerical Study," Micromachines, vol. 11, no. 6, p. 549, 2020, doi: 10.3390/mil 1060549.

[5] V. Carvalho, N. Rodrigues, R. A. Lima, and S. Teixeira, "Numerical simulation of blood pulsatile flow in stenotic coronary arteries: The effect of turbulence modeling and non-Newtonian assumptions," in International Conference on Applied Mathematics \& Computer Science, 2020.

[6] V. Carvalho et al. "In vitro stenotic arteries to perform blood analogues flow visualizations and measurements : a Review," Open Biomed. Eng. J., 2020, under review.

[7] G. Makanda, "A numerical analysis of viscoelastic flow between porous moving walls," WSEAS Trans. Fluid Mech., vol. 14, pp. $15-20,2019$.

[8] A. Bartosik, "Simulations of frictional losses in a turbulent blood flow using three rheological models," WSEAS Trans. Fluid Mech., vol. 15, pp. 131-139, 2020, doi: 10.37394/232013.2020.15.13.

[9] I. Varma and R. Maniyeri, "Numerical study of flow in a centrifugal blower," WSEAS Trans. Fluid Mech., vol. 13, pp. 1-9, 2018.

[10] M. Ahmadi and R. Ansari, "Computational simulation of an artery narrowed by plaque using 3D FSI method: Influence of the plaque angle, non-Newtonian properties of the blood flow and the hyperelastic artery models," Biomed. Phys. Eng. Express, vol. 5, no. 4, p. 45037, 2019, doi: 10.1088/2057-1976/ab323f.

[11] S. C. P. Cheung et al., "Experimental and numerical study on the hemodynamics of stenosed carotid bifurcation," Australas. Phys. Eng. Sci. Med., vol. 33, no. 4, pp. 319-328, 2010, doi: 10.1007/s13246-010-0050-4.

[12] T. Yamaguchi, T. Ishikawa, K. Tsubota, Y. Imai, M. Nakamura, and T. Fukui, "Computational Blood Flow Analysis - New Trends and Methods," J. Biomech. Sci. Eng., vol. 1, no. 1, pp. 2950, 2006, doi: 10.1299/jbse.1.29.

[13] D. F. Young, "Effect of a time-dependent stenosis on flow through a tube," J. Manuf. Sci. Eng. Trans. ASME, vol. 90, no. 2, pp. $248-$ 254, 1968, doi: 10.1115/1.3604621.

[14] E. Doutel, N. Viriato, J. Carneiro, J. B. L. M. Campos, and J. M. Miranda, "Geometrical effects in the hemodynamics of stenotic and non-stenotic left coronary arteries-numerical and in vitro approaches," Int. j. numer. method. biomed. eng., vol. 35, no. 1, pp. 1-18, 2019, doi: 10.1002/cnm.3207.

[15] B. Liu, J. Zheng, R. Bach, and D. Tang, "Influence of model boundary conditions on blood flow patterns in a patient specific stenotic right coronary artery," Biomed. Eng. Online, vol. 14, no. Suppl 1, p. S6, 2015, doi: 10.1186/1475-925X-14-S1-S6.

[16] T. Chaichana, Z. Sun, and J. Jewkes, "Hemodynamic impacts of various types of stenosis inthe left coronary artery bifurcation: A patient-specific analysis," Phys. Medica, vol. 29, no. 5, pp. 447452, 2013, doi: 10.1016/j.ejmp.2013.02.001.

[17] P. K. Siogkas et al., "Patient-specific simulation of coronary artery pressure measurements: An in vivo three-dimensional validation study in humans," Biomed Res. Int., vol. 2015, p. 628416, 2014, doi: $10.1155 / 2015 / 628416$.

[18] L. T. Gaudio, M. V. Caruso, S. De Rosa, C. Indolfi, and G. Fragomeni, "Different Blood Flow Models in Coronary Artery Diseases: Effects on hemodynamic parameters," Proc. Annu. Int. Conf. IEEE Eng. Med. Biol. Soc. EMBS, vol. 2018-July, pp. 31853188, 2018, doi: 10.1109/EMBC.2018.8512917.

[19] M. Dabagh, W. Takabe, P. Jalali, S. White, and H. Jo, "Hemodynamic features in stenosed coronary arteries: CFD analysis based on histological images," J. Appl. Math., vol. 2013, p. 11, 2013, doi: 10.1155/2013/715407.

[20] M. Biglarian et al., "Computational investigation of stenosis in curvature of coronary artery within both dynamic and static models," Comput. Methods Programs Biomed., vol. 185, no. 2020, p. 105170, 2019, doi: 10.1016/j.cmpb.2019.105170. 

"Transient blood flow in elastic coronary arteries with varying degrees of stenosis and dilatations: CFD modelling and parametric study," Comput. Methods Biomech. Biomed. Engin., vol. 18, no. 16, pp. 1835-1845, 2015, doi: 10.1080/10255842.2014.976812.

[22] G. R. Liu and X. Torii, Ryo and Wood, Nigel; Hadjiloizou, Nearchos; Dowsey, Andrew; Wright, Andrew; Hughes, Alun;Davies, Justin; Francis, Darrel; Mayet, Jamil; Yang, Guang;Thom, Simon; Xu, "Fluid-structure interaction analysis of a patient-specific right coronary artery with physiological velocity and pressure waveforms," Commun. Numer. methods Eng., vol. 25 , no. 25 , pp. 565-580, 2009, doi: $10.1002 / \mathrm{cnm}$.

[23] E. Doutel, J. Carneiro, J. B. L. M. Campos, and J. M. Miranda, "Artificial stenoses for computational hemodynamics," Appl. Math. Model., vol. 59, pp. 427-440, 2018, doi: 10.1016/j.apm.2018.01.029.

[24] S. H. Frankel and S. Varghese, "Numerical Modeling of Pulsatile Turbulent Flow in Stenotic," J. Biomech. Eng., vol. 125, no. 4, pp. 445-460, 2003, doi: 10.1115/1.1589774.

[25] J. Banks, "Turbulence Modeling in Three-Dimensional Stenosed Arterial Bifurcations," J. Biomech. Eng., vol. 129, no. 1, pp. 4050, 2007, doi: 10.1115/1.2401182.

[26] A. G. Straatman and J. Ryval, "Two-equation Turbulence Modeling of Pulsatile Flow in a Stenosed Tube," J. Biomech. Eng., vol. 126, no. October 2004, pp. 625-635, 2016, doi: 10.1115/1.1798055.

[27] S. S. Mulani and P. I. Jagad, "Analysis of the Effects of Plaque Deposits on the Blood Flow through Human Artery," Int. Eng. Res. J., vol. 41, no. 2, pp. 2319-3182, 2015.

[28] S. Sriyab, "Mathematical Analysis of Non-Newtonian Blood Flow in Stenosis Narrow Arteries," Comput. Math. Methods Med., vol. 2014, no. December 2014, p. 479152, 2014, doi: $10.1155 / 2014 / 479152$

[29] S. Kamangar et al., "Effect of stenosis on hemodynamics in left coronary artery based on patient-specific CT scan," Biomed. Mater. Eng., vol. 30, no. 4, pp. 463-473, 2019, doi: 10.3233/BME191067.

[30] K. K. L. Wong, J. Wu, G. Liu, W. Huang, and D. Ghista, "Coronary arteries hemodynamics : effect of arterial geometry on hemodynamic parameters causing atherosclerosis," Med. Biol. Eng. Comput., 2020.

[31] X. Chen et al., "Hemodynamics in Coronary Arterial Tree of Serial Stenoses," PLOS \ONE, vol. 11, no. 9, p. e0163715, 2016, doi: 10.1371/journal.pone.0163715

[32] S. I. S. Pinto and J. B. L. M. Campos, "Numerical study of wall shear stress-based descriptors in the human left coronary artery," Comput. Methods Biomech. Biomed. Engin., vol. 19, no. 13, pp. 1443-1455, 2016, doi: 10.1080/10255842.2016.1149575.

[33] A. Fuchs, N. Berg, and L. Witterberg, "Stenosis Indicators Applied to Patient-Specific Renal Arteries without and with Stenosis," Fluids, vol. 4, no. 1, p. 26, 2019, doi: 10.3390/fluids4010026.

[34] V. Carvalho et al., "Hemodynamic study in 3D printed stenotic coronary artery models: experimental validation and transient simulation," Comput. Methods Biomech. Biomed. Eng., 2020.

[35] R. Tabe, F. Ghalichi, S. Hossainpour, and K. Ghasemzadeh, "Laminar-to-turbulence and relaminarization zones detection by simulation of low Reynolds number turbulent blood flow in large stenosed arteries," Biomed. Mater. Eng., vol. 27, no. 2-3, pp. 119 129, 2016, doi: 10.3233/BME-161574.

[36] S. A. Ahmed and D. P. Giddens, "Pulsatile poststenotic flow studies with laser Doppler anemometry," J. Biomech., vol. 17, no. 9, pp. 695-705, 1984, doi: 10.1016/0021-9290(84)90123-4.

[37] K. Rogers, The cardiovascular System, First. Britannica Educational Publishing, 2011

[38] F.-F. Zhou, "Coronary Artery Diameter is Inversely Associated with the Severity of Coronary Lesions in Patients Undergoing Coronary Angiography," Cell. Physiol. Biochem., vol. 43, pp. 1247-1257, 2017, doi: 10.1159/000481765.

[39] M. G. Rabby, A. Razzak, and M. M. Molla, "Pulsatile nonNewtonian blood flow through a model of arterial stenosis," Procedia Eng., vol. 56, pp. 225-231, 2013, doi: 10.1016/j.proeng.2013.03.111.

[40] A. Buradi and A. Mahalingam, "Effect of stenosis severity on wall shear stress based hemodynamic descriptors using multiphase mixture theory," J. Appl. Fluid Mech., vol. 11, no. 6, pp. 14971509, 2018, doi: 10.29252 /jafm.11.06.29062

[41] I. Ansys, "ANSYS Fluent User's Guide." 2013.

[42] W. Versteeg, $\mathrm{H} \mathrm{K}$; Malalasekera, An Introduction to Computational Fluid Dynamics, The finite volume method, Second. Prentice Hall, 2007.

[43] E. Doutel, J. Carneiro, J. B. L. M. Campos, and J. M. Miranda, "Experimental and numerical methodology to analyze flows in a coronary bifurcation," Eur. J. Mech. B/Fluids, vol. 67, pp. 341356, 2018, doi: 10.1016/j.euromechflu.2017.09.009.

[44] M. A. Kabir, M. F. Alam, and M. A. Uddin, "A numerical study on the effects of reynolds number on blood flow with spiral velocity through regular arterial stenosis," Chiang Mai J. Sci., vol. 45 , no. 6 , pp. $2515-2527,2018$.

[45] J. H. Ferziger and M. Peric, Computational Methods for Fluid Dynamics, Third. Springer, 2002.

[46] T. L. Poepping, H. N. Nikolov, R. N. Rankin, M. Lee, and D. W. Holdsworth, "An in vitro system for Doppler ultrasound flow studies in the stenosed carotid artery bifurcation," Ultrasound Med. Biol., vol. 28, no. 4, pp. 495-506, 2002, doi: 10.1016/S03015629(02)00479-9.

[47] P. Kelidis and E. Konstantinidis, "Pulsatile flow through a constricted tube : effect of stenosis morphology on hemodynamic parameters," Comput. Methods Biomech. Biomed. Engin., vol. 21, no. 7, pp. 479-487, 2018, doi: 10.1080/10255842.2018.1481505.

[48] P. Taylor, F. Linge, A. Hye, M. C. Paul, M. A. Hye, and M. C. Paul, "Pulsatile spiral blood flow through arterial stenosis," Comput. Methods Biomech. Biomed. Engin., vol. 17, no. 15, pp. 1727-1737, 2014, doi: 10.1080/10255842.2013.765411.

[49] T. L. Poepping, R. N. Rankin, and D. W. Holdsworth, "Flow patterns in carotid bifurcation models using pulsed doppler ultrasound: Effect of concentric vs. eccentric stenosis on turbulence and recirculation," Ultrasound Med. Biol., vol. 36, no. 7, pp. 1125-1134, 2010, doi: 10.1016/j.ultrasmedbio.2010.02.005.

[50] S. Kefayati, D. W. Holdsworth, and T. L. Poepping, "Turbulence intensity measurements using particle image velocimetry in diseased carotid artery models: Effect of stenosis severity, plaque eccentricity, and ulceration," J. Biomech., vol. 47, no. 1, pp. 253263, 2014, doi: 10.1016/j.jbiomech.2013.09.007.

[51] A. L. DiCarlo, D. W. Holdsworth, and T. L. Poepping, "Study of the effect of stenosis severity and non-Newtonian viscosity on multidirectional wall shear stress and flow disturbances in the carotid artery using particle image velocimetry," Med. Eng. Phys., vol. 65, pp. 8-23, 2018, doi: 10.1016/j.medengphy.2018.12.023.

\section{Creative Commons Attribution License 4.0} (Attribution 4.0 International, CC BY 4.0)

This article is published under the terms of the Creative Commons Attribution License 4.0 https://creativecommons.org/licenses/by/4.0/deed.en US 